\title{
Unraveling the mechanism of action of thiazolidinediones
}

\author{
C. Ronald Kahn, Lihong Chen, and Shmuel E. Cohen
}

Joslin Diabetes Center and Harvard Medical School, Boston, Massachusetts, USA

Address correspondence to: C. Ronald Kahn, Joslin Diabetes Center, One Joslin Place, Boston, Massachusetts 02215, USA.

Phone: (617) 732-2635; Fax: (617) 732-2593; E-mail: c.ronald.kahn@joslin.harvard.edu.

Thiazolidinediones (TZDs) are a new class of antidiabetic agents and include three compounds that have come to clinical use - troglitazone $\left(\right.$ Rezulin $\left.^{\circledR}\right)$, rosiglitazone (Avandia $\left.{ }^{\circledR}\right)$, and pioglitazone $\left(\right.$ Actos $\left.^{\circledR}\right)$ - as well as several others that have been limited to pre-clinical study. TZDs were initially discovered by screening compounds for a hypoglycemic action in the $o b / o b$ mouse (1), and subsequently they were shown to improve insulin action in a variety of obese and diabetic animal models with insulin resistance (2). In these model systems, TZDs reduce plasma glucose and insulin levels and improve some of the abnormalities of lipid metabolism. Consistent with animal studies, clinical studies have shown that treatment of type 2 diabetic patients with TZDs can lower serum glucose and insulin levels, increase peripheral glucose uptake, and decrease triglyceride levels (3). In euglycemic clamp studies, this is associated with an increase in insulin sensitivity in peripheral tissues (mainly represented by muscle in clamp studies), with relatively little effect on hepatic glucose output (4).

Insights into the molecular mechanism of action of TZDs came from studies that demonstrated that these agents increase transcription of certain genes in adipose tissue $(5,6)$. The recognition that the promoter region of one of the genes, the fat-specific gene $a P 2$, binds a transcription factor identified as PPAR $\gamma(7)$ led Spiegelman, Kletzien, and Kliewer (6-9) to propose that TZDs exert their effects by activating this nuclear receptor. Due to alternative splicing and alternative promoter usage, there are two isoforms of PPAR $\gamma$ ( 1 and 2 ), and both are predominantly expressed in fat cells (10). Subsequent studies have shown an excellent correlation between the hypoglycemic action of the TZDs and their affinity for PPAR $\gamma$. Regulation of gene expression via PPAR $\gamma$ is tissue-specific (10), and although its role in adipocyte differentiation is well documented (10), the normal functions of PPAR $\gamma$ remain unclear. Complete elimination of a functional PPAR $\gamma$ gene results in embryonic lethality, whereas PPAR heterozygote knockout mice with a $50 \%$ reduction in PPAR $\gamma$ expression exhibit increased basal insulin sensitivity and resistance to high-fat diet-induced insulin resistance (11).

Despite the evidence that TZDs act by binding to PPAR $\gamma$ and can improve different insulin-resistant states in both human and animal studies, the exact mechanism and site of action of the TZDs are still unclear. Type 2 diabetes is a complex metabolic syndrome with alterations in glucose, lipid, and protein metabolism (12). Hyperglycemia in type 2 diabetes is due to a decrease in insulin action to stimulate glucose uptake in peripheral tissues, such as muscle and fat, as well as unsuppressed hepatic glucose output and relative hypoinsulinemia. Traditional thinking suggests that the defect in muscle glucose uptake accounts for most of the postprandial hyperglycemia, the increase in fasting glucose is due to the increased hepatic glucose output, and the lipid abnormalities are due to a combination of defects in fat and liver (12). However, recent studies in mice in which insulin resistance has been created in individual tissues by conditional gene knockout suggest that these classical views may have been oversimplified (13-15). Thus, the muscle-specific insulin receptor knockout mouse exhibits hypertriglyceridemia and increased FFAs (14), the liver-specific knockout exhibits only mild hyperglycemia and lower triglycerides (15), the fat-specific knockout has normal glucose tolerance (M.D. Michael and C.R. Kahn, unpublished observations), and the $\beta$ cell-specific knockout of the insulin receptor produces a defect in glucose-stimulated insulin release (13).
The adipocyte has a special role in the overall pathogenesis of insulin resistance, since most type 2 diabetes is associated with obesity, and adipocytes from obese animals have been shown to exhibit a number of properties that may be associated with insulin resistance (Figure 1, left panel). These include the fact that obesity is associated with hypertrophy of the adipocyte itself, leading to insulin resistance as well as overproduction of FFAs, TNF- $\alpha$, and leptin, all of which have been shown to have effects that induce insulin resistance in other peripheral tissues $(11,16)$. Therefore, the key question is, are the effects of TZDs mediated exclusively through direct action on the adipocyte, with indirect effects to improve insulin sensitivity in other tissues, or are other tissues also important targets of the TZD agents? Since PPAR $\gamma$ is most highly expressed in adipose tissue, and since TZDs have been shown to regulate gene expression in fat, most speculation has focused on the adipocyte as the site of primary response. However, low levels of PPAR $\gamma$ receptor mRNA can be observed in many tissues, leaving this issue unresolved.

In normal humans, up to $80 \%$ of insulin-stimulated glucose disposal occurs in skeletal muscle, and this is a major site of insulin resistance in type 2 diabetes (2). Studies using euglycemic, hyperinsulinemic clamps have demonstrated that TZD treatment is associated with improved insulin sensitivity and increased insulin-stimulated glucose disposal in muscle, with little effect on hepatic glucose output (4). TZDs have also been shown to increase insulin signaling in vitro using muscle culture cells (17). On the other hand, TZDs have been shown to lower levels of circulating FFAs that could contribute to insulin resistance (18). TZDs also stimulate adipocyte differentiation, generating small adipocytes that are more insulin-sensitive than large adipocytes (19) (Figure 1, right panel). 
Recently, two groups of investigators have generated transgenic models of lipoatrophy that provide an opportunity to study the role of adipose tissue in the antidiabetic action of TZDs $(20,21)$. One of the lipoatrophic models was based on use of an attenuated form of the diphtheria toxin A chain (DTA) under the control of the adipocyte-specific aP2 promoter to create a cell-specific toxigene (20). By 8-10 months of age, these mice have no visible subcutaneous or intra-abdominal adipose tissue and have severe diabetes with hyperinsulinemia, hyperglycemia, hyperlipidemia, and fatty liver $(20,22)$. When these animals were treated with troglitazone for 5 weeks, their insulin sensitivity was improved, glucose tolerance normalized, and serum insulin, cholesterol, triglyceride, and FFA levels were dramatically reduced, despite the absence of fat (22). These data suggest that TZDs do not require significant levels of adipose tissue to improve insulin sensitivity and that there is probably important and direct major action of troglitazone on other tissues, most likely skeletal muscle.

In a recent issue of the JCI, Chao et al. further explored the role of adipose tissue in the mechanism of action of TZDs, using an even more severe transgenic lipoatrophic mouse model, the A-ZIP/F1 mouse (23). These mice express a dominant-negative protein, A-ZIP/F, under the control of the adipose-specific $a P 2$ enhancer/promoter (21). This protein inhibits DNA binding and function of the B-ZIP transcription factors of both the C/EBP and JUN families, producing a severe disruption of adipocyte growth and differentiation. A-ZIP/F-1 mice have no visible white adipose tissue (WAT) and significantly reduced brown adipose tissue throughout life, and adults develop hyperglycemia with polyphagia, polyuria, and polydipsia, severe insulin resistance, and hyperlipidemia $(21,23)$. Also, as with aP2/DTA animals (22), treatment with rosiglitazone or troglitazone lowers serum triglyceride and FFA levels to normal in A-ZIP/F-1 mice, further supporting the finding that TZDs are able to correct hyperlipidemia in mice lacking adipose tissue. However, treatment of these mice with TZDs at concentrations that were effective in lowering glucose in aP2/DTA and $o b / o b$ mice failed to reduce the serum glucose and insulin levels and to improve glucose tolerance in the A-ZIP/F-1 mice (23). This leads the authors to conclude that while the effects of TZDs on lipid metabolism do not depend on the action of these drugs on fat, their actions on glucose metabolism require the presence of adipose tissue.

Why the effect of TZDs is different in these two lipoatrophic diabetic animal models is unclear. The models are of different mechanism, severity, and duration of lipoatrophy and in mice of different genetic background. In some humans with lipoatrophic diabetes, treatment with troglitazone results in remarkable improvement in hyperglycemia, as well as a significant decrease in triglyceride and FFA levels (24). This improvement is accompanied by the redistribution of, and a small but significant increase in, adipose tissue (24). However, it is unlikely that the increase in glucose disposal in aP2/DTA mice could be accounted for by the small amount of residual fat in this model. It is possible that TZDs stimulate this residual adipose tissue, which, in turn, conveys some signals (perhaps through leptin or TNF- $\alpha$ ) to skeletal muscle and improves insulin sensitivity (Figure 1, right panel). It is also possible that the direct effect of TZDs on skeletal muscle requires the presence of signals from other tissues, including WAT. These signals could be missing in the AZIP/F-1 mice, which have a complete absence of WAT. This hypothesis is also supported by the study of surgical implantation of adipose tissue in A$\mathrm{ZIP} / \mathrm{F}-1$ mice $(23,25,26)$. Both in vivo and in vitro experiments demonstrated that skeletal muscle from A-ZIP/F-1 mice has impaired response to insulin stimulation, and fat transplantation normalized glucose uptake and reversed insulin resistance in skeletal muscle from these animals. Interestingly, TZDs did not affect fat-transplanted A-ZIP/F1 mice (23), perhaps because these mice already enjoy improved insulin sensitiv-
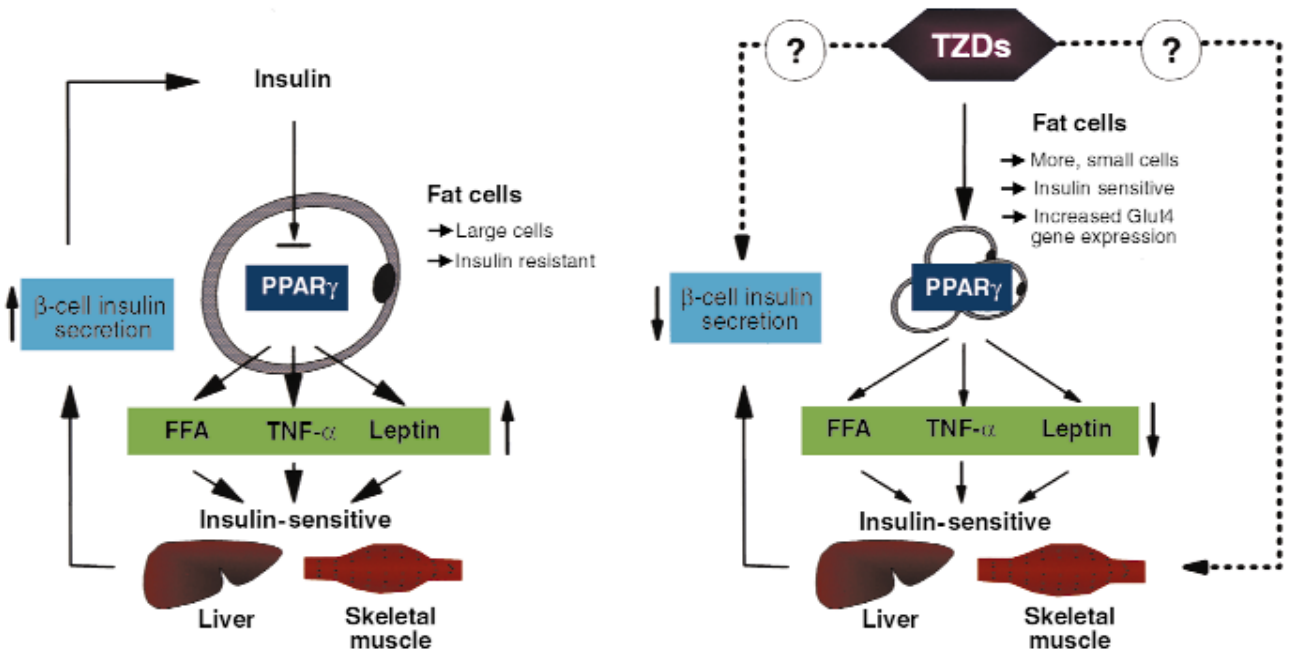

Figure 1

Left panel: Fat cells in the insulin resistance of type 2 diabetes are larger than in the normal subjects, with increased production of FFA, TNF- $\alpha$, and leptin. This leads to insulin resistance in the liver and skeletal muscle, resulting in a need for increased peripheral insulin, which is generated by the $\beta$ cells in the pancreas. Right panel: TZDs stimulate adipocyte differentiation, preferentially generating more numerous, smaller adipocytes. These small adipocytes are more insulin-sensitive, producing less FFA, TNF- $\alpha$, and leptin. Therefore, insulin is more effective and there is less need for the $\beta$ cells to increase their secretion of insulin. 
ity as a result of the restoration of some WAT. Future studies using genetic techniques to create tissue-specific knockout of PPAR $\gamma$ in individual insulin-sensitive tissues, such as skeletal muscle, fat, and liver, as well as in pancreatic $\beta$ cells, are needed; these animal models should answer not only the question of the primary site of the antidiabetic effect of TZDs, but also perhaps of the role of this receptor in normal function of each of these tissues.

1. Fujiwara, T., Yoshioka, S., Yoshioka, T., Ushiyama, I., and Horikoshi, H. 1988. Characterization of new oral antidiabetic agent CS-045. Studies in $\mathrm{KK}$ and ob/ob mice and Zucker fatty rats. Diabetes. 37:1549-1558.

2. Olefsky, J.M. 2000. Treatment of insulin resistance with peroxisome proliferator-activated receptor $\gamma$ agonists. J. Clin. Invest. 106:467-472.

3. Saltiel, A.R., and Olefsky, J.M. 1996. Thiazolidinediones in the treatment of insulin resistance and type II diabetes. Diabetes. 45:1661-1669.

4. Inzucchi, S.E., et al. 1998. Efficacy and metabolic effects of metformin and troglitazone in type II diabetes mellitus. N. Engl. J. Med. 338:867-872.

5. Ibrahimi, A., et al. 1994. Evidence for a common mechanism of action for fatty acids and thiazolidinedione antidiabetic agents on gene expression in preadipose cells. Mol. Pharmacol. 46:1070-1076.

6. Kletzien, R.F., Foellmi, L.A., Harris, P.K., Wyse, B.M., and Clarke, S.D. 1992. Adipocyte fatty acid-binding protein: regulation of gene expression in vivo and in vitro by an insulin-sensitizing agent. Mol. Pharmacol. 42:558-562.

7. Tontonoz, P., Hu, E., Graves, R.A., Budavari, A.I., and Spiegelman, B.M. 1994. mPPAR gamma 2: tissue-specific regulator of an adipocyte enhancer. Genes Dev. 8:1224-1234.

8. Forman, B.M., et al. 1995. 15-Deoxy-delta 12, 14 prostaglandin $\mathrm{J} 2$ is a ligand for the adipocyte determination factor PPAR gamma. Cell. 83:803-812.

9. Lehmann, J.M., et al. 1995. An antidiabetic thiazolidinedione is a high affinity ligand for peroxisome proliferator-activated receptor gamma (PPAR gamma). J. Biol. Chem. 270:12953-12956.

10. Rosen, E.D., Walkey, C.J., Puigserver, P., and Spiegelman, B.M. 2000. Transcriptional regulation of adipogenesis. Genes Dev. 14:1293-1307.

11. Kadowaki, T. 2000. Insights into insulin resistance and type 2 diabetes from knockout mouse models. J. Clin. Invest. 106:459-465.

12. Olefsky, J.M. 1995. Diabetes mellitus (type II) etiology and pathogenesis. In Endocrinology. Volume 2. L.J. DeGroot, editor. W.B. Saunders Co. Philadelphia, Pennsylvania, USA. 1436-1463.

13. Kulkarni, R.N., et al. 1999. Tissue-specific knockout of the insulin receptor in pancreatic beta cells creates an insulin secretory defect similar to that in type 2 diabetes. Cell. 96:329-339.

14. Bruning, J.C., et al. 1998. A muscle-specific insulin receptor knockout exhibits features of the metabolic syndrome of NIDDM without altering glucose tolerance. Mol. Cell. 2:559-569.

15. Michael, M.D., et al. 2000. Loss of insulin signaling in hepatocytes leads to severe insulin resistance and progressive hepatic dysfunction. Mol. Cell. 6:87-97.

16. Smith, U., et al. 1999. Insulin signaling and action in fat cells: associations with insulin resistance and type 2 diabetes. Ann. NY Acad. Sci. 892:119-126.

17. Ciaraldi, T.P., Gilmore, A., Olefsky, J.M., Goldberg, M., and Heidenreich, K.A. 1990. In vitro studies on the action of CS-045, a new antidiabetic agent. Metabolism. 39:1056-1062.

18. Bergman, R.N., and Ader, M. 2000. Free fatty acids and pathogenesis of type 2 diabetes mellitus. Trends Endocrinol. Metab. 11:351-356.

19. Okuno, A., et al. 1998. Troglitazone increases the number of small adipocytes without the change of white adipose tissue mass in obese Zucker rats. J. Clin. Invest. 101:1354-1361.

20. Ross, S.R., Graves, R.A., and Spiegelman, B.M. 1993. Targeted expression of a toxin gene to adipose tissue: transgenic mice resistant to obesity. Genes Dev. 7:1318-1324.

21. Moitra, J., et al. 1998. Life without white fat: a transgenic mouse. Genes Dev. 12:3168-3181.

22. Burant, C.F., et al. 1997. Troglitazone action is independent of adipose tissue. J. Clin. Invest. 100:2900-2908.

23. Chao, L., et al. 2000. Adipose tissue is required for the antidiabetic, but not for the hypolipidemic, effect of thiazolidinediones. J. Clin. Invest. 106:1221-1228.

24. Arioglu, E., et al. 2000. Efficacy and safety of troglitazone in the treatment of lipodystrophy syndromes. Ann. Intern. Med. 133:263-274.

25. Kim, J.K., Gavrilova, O., Chen, Y., Reitman, M.L., and Shulman, G.I. 2000. Mechanism of insulin resistance in A-ZIP/F-1 fatless mice. J. Biol. Chem. 275:8456-8460.

26. Gavrilova, O., et al. 2000. Surgical implantation of adipose tissue reverses diabetes in lipoatrophic mice. J. Clin. Invest. 105:271-278. 